\title{
Pierre Gy: How I discovered the Theory of Sampling and got to know a great scientist
}

Pentti Minkkinen

\section{Introduction: first encounters with sampling problems}

graduated from Helsinki University of

Technology, Department of Chem-

istry in 1969. In analytical chemistry

courses at that time hardly anything was taught about sampling, besides the phrases "the result is no better than the sample" or "the sample must be representative". But "how to" achieve that was not explained. Coning and quartering was the only recommended sample size reduction method. Sadly, this is still the case in the majority of universities offering chemistry. However, in our final exam on analytical chemistry there was the following question: "You receive a container containing liquid, some slurry and a lump of metal (size about $1 \mathrm{~kg}$ ) with a request to estimate the average concentration of the metallic constituents of the sample. How do you proceed with the analysis?" In my answer I proposed that if it is possible to dissolve the whole sample a good estimate of the average concentrations is obtained by analysing the resulting solution. If that was not feasible, the liquid, slurry and the metal piece should be separated and analysed separately. The metal should be sampled so that several subsamples should be drilled from it from different sides. Many years later I learned that I had described the principle of the composite sampling mode.

I met my first real-world sampling problem in 1966, when still a student and still ignorant of any theory of sampling. I had a summer job as a shift foreman in a company producing granulated super phosphate. The company was exporting this product to a customer who had a very tight quality criterion for the phosphate content. There appeared to be a quality problem. Samples taken from the stream coming from the granulator gave phosphate values that were below the specification, whereas samples taken at the storage room gave results meeting the specifications. I looked at the problem. Samples were taken manually from the middle of the falling stream coming from the granulator with a cup having a round opening and volume of about $1 / 2$ litre. I could actually observe that the fine and coarse particles in the stream were segregated and were being sampled unevenly from the middle of the stream. So I suggested that the phosphate content of the fine and coarse part were analysed separately and the result indeed showed a difference in phosphate content explaining the observed deviations. So I designed a sample cutter with a rectangle opening to be used manually as a cross-stream sampler. Samples taken with that method gave results which met the specifications.

From June 1973 to July 1974 I was working as an Associate Expert (Analytical Chemist) in a United Nation Development Program (UNDP) Mineral Exploration project in Turkey. In this project a quality control programme based on duplicate sampling was in use. This programme was used to monitor the uncertainty of both the field sampling and laboratory results. In the field, duplicate soil and sediment samples approximately $2 \mathrm{~m}$ apart were regularly taken and, in the laboratory, duplicate analytical samples were prepared and analysed from the laboratory samples. Reference samples and inter laboratory comparisons were also used for control purposes. The results were scrutinised but not systematically analysed. In my opinion the methods then proposed to analyse such data were not adequate for their intended use. The main problem was that the exploration samples had wide ranges of concentrations and quite obviously the standard deviations both of the samples and analyses were dependent on concentration. Consequently, the variances were dependent on the concentration level which made the assumption on normal distribution invalid and the pooling of information obtained from the duplicates difficult and very doubtful. This had to be taken into account in the subsequent data analyses.
The Anatolian plateau is covered by snow in winter for a few months. As consequence, very few exploration samples arrived in the laboratory during this time. That gave me time from routine work to think if the existing quality control data could be used more effectively than up to then. I noticed that while absolute standard deviation was strongly dependent on concentration, the relative standard deviations were practically constant over a concentration range covering several order-of-magnitudes starting from concentrations approximately five times above detection limits of the analytical methods. At these concentrations the relative standard deviation estimates calculated for each duplicate and having just one degree of freedom could be pooled into one realistic total relative standard deviation estimate with degrees of freedom equal to the number of the duplicates. I wrote an internal report proposing this method to be used as quality control method both for the laboratory and field sampling. The report was well accepted with recommendation to adopt it in other UNDP projects. After working in another UNDP project in Egypt, I returned in August 1975 back to Finland and continued my graduate studies at Helsinki University of Technology where I wrote my graduate thesis on the use of duplicates in routine quality control. Figures 1-2 show some examples of how the method can be used in routine control. Figure 1 shows the results of atomic absorption spectrometric determinations of lead from the laboratory duplicates of soil samples covering a concentration range from $60 \mathrm{ppm}$ to $17,000 \mathrm{ppm}$. As can be seen, the absolute standard deviation has a strong concentration dependence whereas the relative standard deviation now shows systematic concentration dependence. The pooled relative standard deviation was $7.8 \%$.

The uncertainty of the primary sampling was tested by taking field samples approximately $2 \mathrm{~m}$ apart and submitting both to 


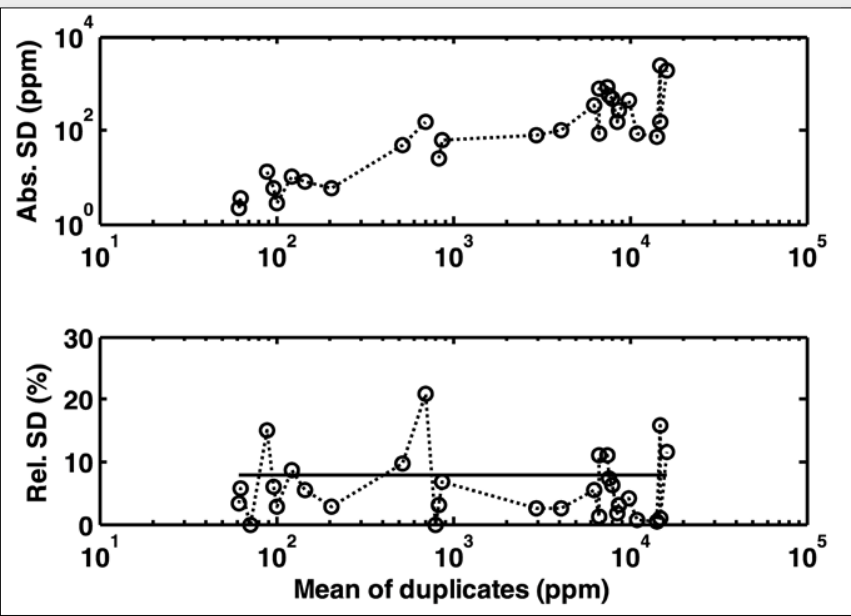

Figure 1. Duplicate Pb analyses from geological soil samples. Absolute standard deviations from the duplicates (upper panel) show strong concentration dependence. Relative standard deviation estimates (lower panel) are independent on concentration, thus the pooled value (horizontal line), $s_{\text {, }}$ (pooled $)=7.8 \%$, can be used over this whole concentration range as the estimate of the standard uncertainty, with a coverage factor 2, giving the expanded relative uncertainty value $U_{r}=15.6 \%$
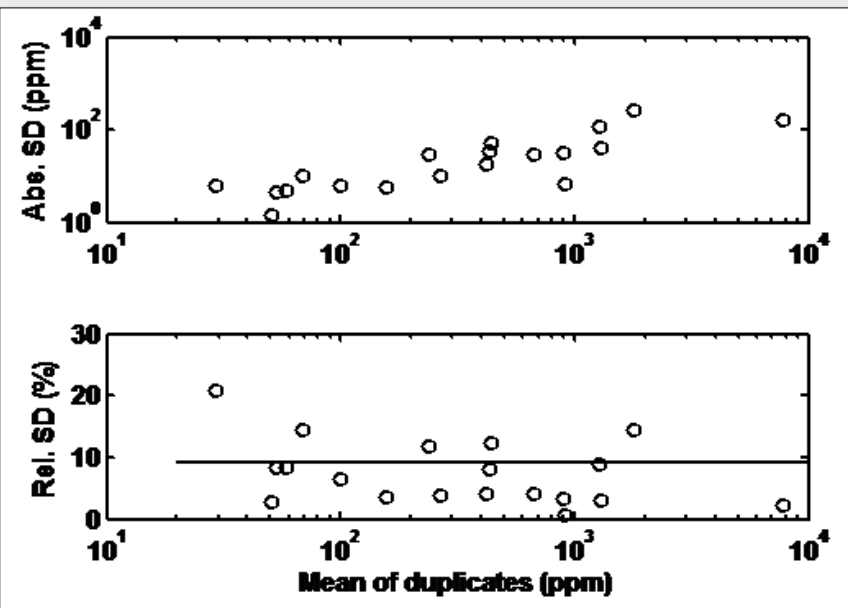

Figure 2. Duplicate Pb analyses from field duplicates taken about $2 \mathrm{~m}$ apart. Absolute standard deviations from the duplicates (upper panel) show strong concentration dependence. Relative standard deviation estimates (lower panel) are independent on concentration, thus the pooled value (horizontal line), $s_{\text {, }}$ (pooled) $=9.1 \%$, can be used over this whole concentration range as the estimate of total standard uncertainty, with the coverage factor 2, giving an expanded relative uncertainty value $U_{r}=18.2 \%$. analysis in laboratory. These results give the sum of the short range field sampling variance in addition to the variance of the laboratory procedure. Figure 2 shows the absolute and relative standard deviations calculated for each sample pair. As in Figure 1 , the absolute standard deviation is strongly dependent on concentration, but the relative standard deviation is obviously independent on the tested concentration range. The pooled relative standard deviation estimate of the whole data set is here $9.1 \%$. This value does not differ significantly from the laboratory duplicate results showing that laboratory sample preparation (sieving, subsampling) and analysis contribute the major part of the total variance. In retrospect, the low primary sampling error can be explained. Soil samples were dried and then sieved. The below 80 mesh $(0.18 \mathrm{~mm})$ fraction was used as the laboratory sample. From it $500 \mathrm{mg}$ or $200 \mathrm{mg}$ analytical samples were taken with a spatula, i.e. as grab samples. It is obvious, of course, that in this case segregation and fundamental sampling errors are high. For instance, at 500 ppm $\mathrm{Pb}$ (if lead is present as galena) the relative standard deviation of the fundamental sampling error of a $200 \mathrm{mg}$ sample is about 6\% At that time nobody had any idea yet how to calculate it. Anyway, these results showed that the sampling analytical methods were adequate for mapping interesting areas.
In the third example (Figure 3) lead results from the central laboratory and the field laboratory were compared by analysing the same samples in both laboratories. In the central laboratory the metals from the geological field samples were extracted by aqua regia and in the field laboratory with nitric acid. By using the central laboratory results as reference values, the absolute and relative differences from the reference values are plotted as function of the reference results. As can be seen, the field laboratory results are negatively biased at low concentration range and positively at high concentrations. The relative differences can be modelled with

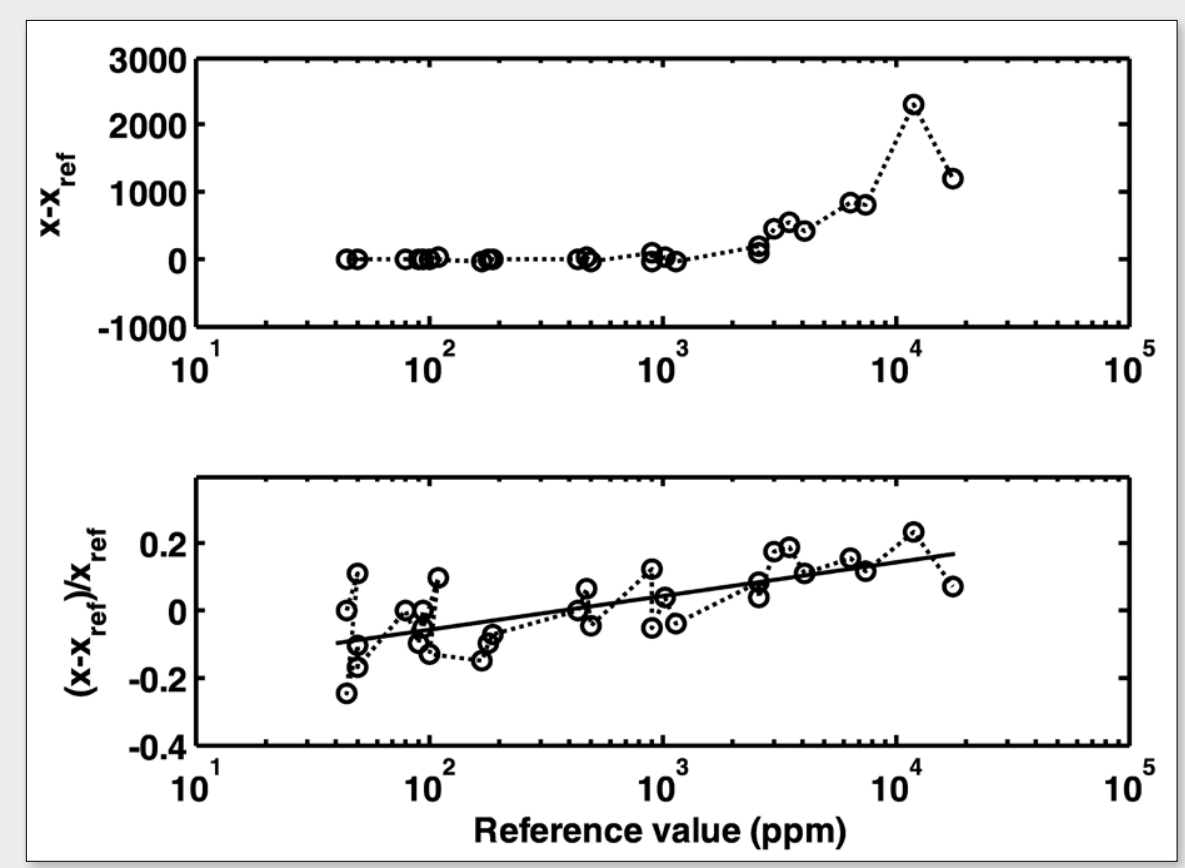

Figure 3. Comparison of two methods of lead analysis from geological soil samples, based on absolute (upper panel) and relative (lower panel) deviations from the reference method (aqua regia). The relative differences of the methods can be modelled with linear regression as function of the concentration. The residual standard deviation from the regression line is $0.086(=8.6 \%)$. 
linear regression and, if necessary, the field laboratory results can be converted to be equivalent with the central laboratory results. The traditional t-test of duplicates fails in this case to detect any significant differences with these two sets of the results - also an interesting result, and still often used today to analyse data sets similar to this example.

\section{Getting to know about Pierre Gy's Theory of Sampling}

In 1976 I got first a temporary, and later a permanent job, as Associate Professor in inorganic and analytical chemistry at the newly founded regional Lappeenranta University of Technology (LUT). The next year the first students were to start chemical engineering studies. Dr Seppo Wilska, Professor of Chemical Technology, was the first full professor appointed and the head of the new department of Chemical Technology. He also planned the curriculum of the department. He had planned that I should give a one semester course in process analytical chemistry to third year students. He stated that: “... in process analytical chemistry there is a fundamental lack of knowledge, namely on the importance of sampling. Nobody seems to be interested in sample quality and nobody is teaching this subject". Since I had shown interest in analytical quality control, he suggested that I should also start teaching sampling. More importantly, he indicated that there was a man who had been working with sampling and had developed a very useful theory, and equations, with clever approximations allowing the estimation of actual sampling variance from known material properties. This man, needless to say here, turned out to be Pierre Gy. Professor Wilska also gave me Pierre's first publication, written in German. In that article Pierre presented his famous equation for estimating the variance of the fundamental sampling error. I presented this equation and its applications in my first lecture series about sampling. When I received Pierre Gy's 1982 book: Sampling of Particulate Materials - Theory and Practice (second revised edition) the beauty of TOS really opened up to me. That was the first presentation that logically covered the whole sampling process: design and operation principles of sampling equipment, sources of sampling errors and the data analysis needed in estimating the total sampling uncertainty. Completely new to me was also that the three different sampling modes, random, stratified and systematic, produce different variances of the lot mean, even if exactly the same number of primary samples are taken and analysed from the same lot. It took some mental wrestling to believe this, so deeply had the normal distribution rooted in my mind. Many guidelines I had read so far gave the advice always to use random sampling if there is systematic variation in the sampling target. Pierre Gy showed that the random mode leads to higher variance of the mean than the two other approaches, periodic process being the exception, if the sampling frequency is too low. The expected experimental variance is the same and independent of the sampling mode, however. Only in estimating the variance of the mean, correction for the autocorrelation due to the systematic variation depends on sampling mode. Gy also presented a practical method for estimating the correction needed for onedimensional lots: variographic analysis. Later I reviewed, for Chemometrics and Intelligent Laboratory Systems, Piere Gy's second book written in English (1992). Reading these books it became obvious that the fundamental assumption on randomness is hardly ever justified in planning primary sampling, and more importantly, leads to inflated and too expensive sampling plans.

Campbell et al., "Sampling, sample preparation, and sampling plans for foodstuffs for mycotoxin analysis", Pure and Applied Chemistry 58 (1986) described an experimental study where the variances of the primary sampling and laboratory measurements were estimated as function of the mean concentration of aflatoxins in peanuts lots. That article gave $112 \mathrm{mg} \mathrm{kg}^{-1}$ for the average aflatoxin concentration in mouldy peanuts. I had an idea to compare these experimental results to the results obtained by using Pierre Gy's theory. To have the other necessary material properties I drove to the nearest shop and bought $100 \mathrm{~g}$ of peanuts, came back to my laboratory and in half an hour I had estimates for the necessary material properties: nominal particle size, density, size distribution and shape factors. Gy's famous equation gave a variance estimate that was about half of the experimental value. As mould-producing aflatoxin is usually present in small pockets irregularly distributed spatially in peanut lots, the grouping and segregation error most certainly also has to be taken into account. According to Gy, the grouping and segregation variance can be equal to fundamental sampling error variance. When account is taken of this theoretical result, which cost only the $100 \mathrm{~g}$ of peanuts and half an hour's work, my small empirical study gave almost exactly the same result as the massive experimental study of Campbell et al., which must have cost at least tens of thousands dollars or more. That was enough to convince me of the value of Pierre Gy's Theory of Sampling (TOS).

\section{Meeting Pierre Gy personally}

To make calculations easier I had written a small computer program for different applications of Gy's fundamental sampling error equation. I demonstrated it at one analytical chemists' meeting. One of the commercial participants, who sold analytical instruments, stated that he absolutely must have this program. Often his customers complained that his analyser was not reliable, when, in his opinion the analyser was working properly-but he suspected that the real culprit was poor sampling and sample preparation. With my program, he believed he would be able to prove this. So I commercialised this little program and called it SAMPEX, with the help of friend who had a small company producing programs for process monitoring and other process applications. With audacity I sent this program to Pierre Gy for his comments. I was very pleased when he quickly answered and told that he liked it ...

The first time I had a chance to meet Pierre personally was in Graz 1994 at the EURACHEM Workshop, where he was an invited speaker. Besides sampling and analytical quality control I was also teaching chemometrics, applying this multivariate data analysis approach extensively in my own research. But my fellow chemometricians were mainly, indeed overwhelmingly ONLY, interested in the multivariate methods themselves (PCA and PLS) and their applications to solve complicated chemical problems. Also in this community there was little, if any, interest in data quality. Personally I had an opinion that TOS is, or at least it should be, a branch of chemometrics. I presented contributions related to TOS 


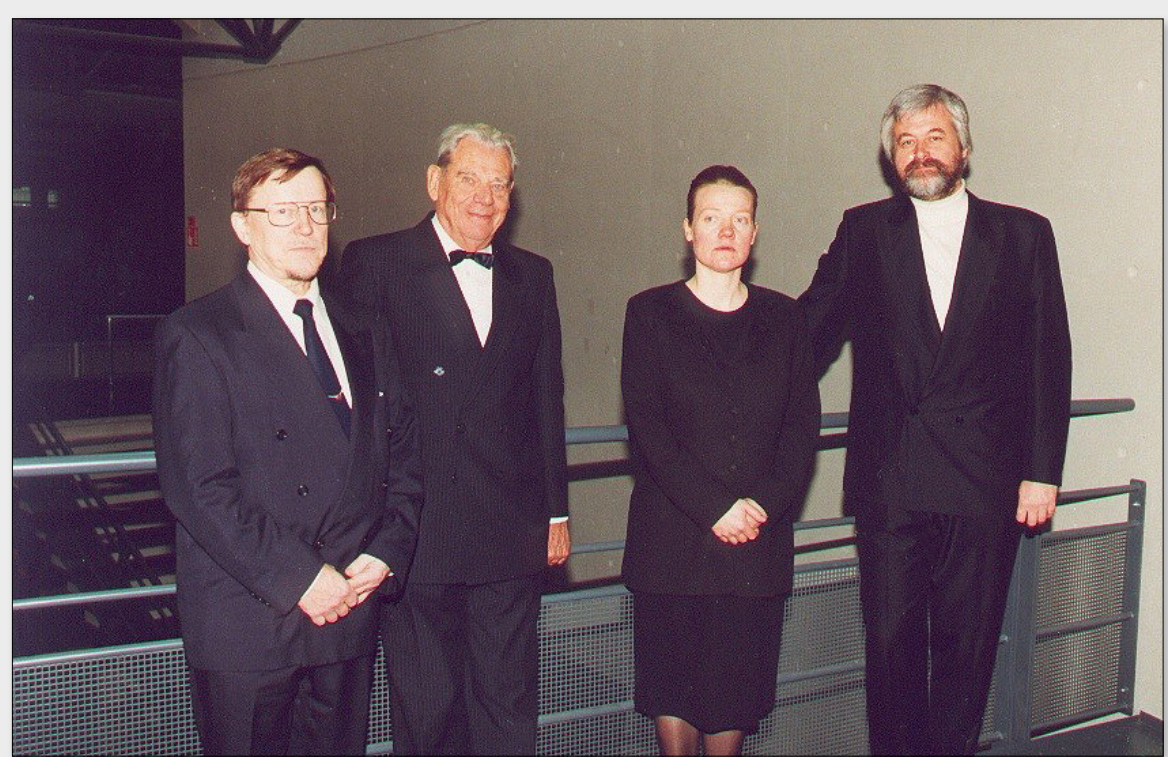

Figure 4. Photo taken at Riitta Heikka's dissertation defence. Left to right: Pentti Minkkinen, Pierre Gy, Riitta Heikka and Kim H. Esbensen.

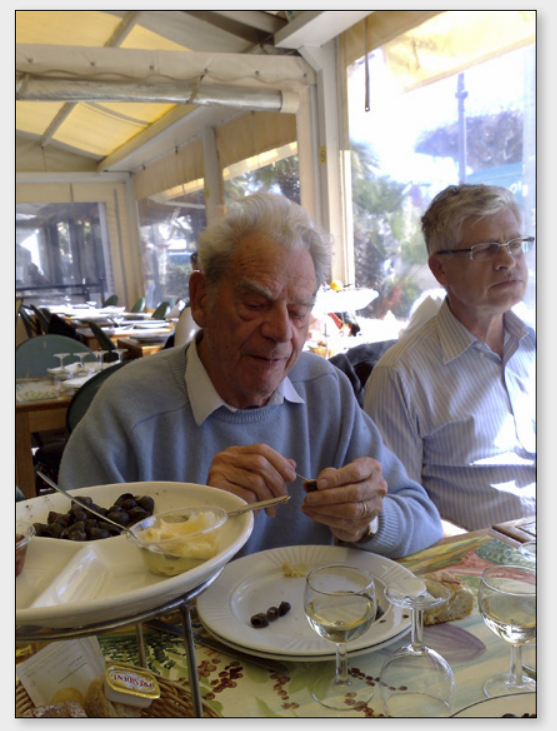

Pierre Gy in Cannes.

in several chemometric conferences and in private discussions over many years. The response from my friends usually consisted in heads nodding politely with comments like: "very interesting". However, nothing more followed.

That probably would still be the case, even today, were it not for Pierre Gy's personal involvement. In the late 1990s I had a graduate student, Riitta Heikka, whose doctoral thesis was concerned both with applications of chemometrics as well as sampling. She defended her thesis late in 1998. I invited two opponents to the dissertation: an already seasoned chemometrician, Professor Kim H. Esbensen and an even more seasoned sampling expert, Dr Pierre Gy (Figure 4). I had for some time had some hope that surely my friend Kim would be able to understand the importance of TOS, alas up to that time to very little avail. But when these two gentlemen of science met, officiated together and, probably most influentially, spent an entire lay-over weekend in the same hotel in my regional city of Lappeenranta where little of the cultural activities of cities like Copenhagen and Cannes were to be found (and temperatures were well below zero), Kim could not resist any more. He saw the light, converted on the spot and became a zealous disciple of Pierre Gy. That is probably my greatest contribution to promote TOS! The rest is history. Kim took the beacon and the result is a successful series of sampling conferences, this new communication platform devoted to TOS, a standard based on TOS and many publications.

\section{The next 15 years}

After Riitta Heikka's dissertation I had occasion to meet Pierre Gy many times. It was always a distinct pleasure. The last time was as part of the small delegation, who after the WCSB7 in Bordeaux 2015 , organised a meeting at his caretaking institution. He was in good mood and happy to receive visitors.

At the Graz meeting he told in his lecture that for tens of years during his consultancy all over the World, he was always asking his clients who in their company were responsible for proper sampling. The usual answer was: "Err, somebody else, not in my department". So far, he said, he had been unable to identify who that particular somebody was. He also challenged analytical chemists to take the lead in promoting TOS. Not all that much has changed in the intervening 20 years or so-or maybe it has ....

I owe Pierre the greatest debt of gratitude. At least half of my professional success both in academia and private consultancy has resulted directly from TOS. That has given me a chance to get new friends, see new interesting places and the chance to work with interesting problems and talented people. I am very proud to have been honoured as a recipient of the Pierre Gy Sampling Gold Medal. I am in fact the only recipient of both the PGSGM and the Herman Wold Gold medal in Chemometrics.

Pierre once told me that he was afraid that after his time was up, TOS would also go into oblivion. He was disappointed how slowly the analytical community reacted to his challenge, and ditto universities in accepting TOS into their curricula.

Friend Pierre, I assure your soul should be at rest, and in peace. You, and the TOS will never be forgotten. You will live in our memories and the acceptance of your Theory of Sampling will, eventually no matter however slowly in appearance, win ever wider acceptance and respect. We are all dedicated to work on tirelessly for this .... 